Bull. Austral. Math. Soc.

Vol. 46 (1992) [245-261]

\title{
ON PERIODIC SOLUTIONS OF A SEMILINEAR HYPERBOLIC PARABOLIC EQUATION
}

\section{Mitsuhiro Nakao and Hisako Kato}

Uniqueness and regularity of periodic solutions to the semilinear dissipative wave equation with small parameter $\varepsilon>0$,

$$
\varepsilon u_{t z}-\Delta u+u_{t}+g(u)=f(x, t) \text { on } \Omega \times R \text { and }\left.u\right|_{\theta \Omega}=0, \quad \Omega \subset R^{N},
$$

are investigated when $g(u)$ has a certain 'critical' nonlinearity.

\section{INTRODUCTION}

Let $\Omega$ be a bounded domain in the $N$-dimensional Euclidean space $R^{N}$ with smooth boundary $\partial \Omega$ and let us consider the periodicity problem for the semilinear wave equation with a dissipation:

$$
\begin{cases}u_{t t}-\Delta u+\nu u_{t}+g(u)=f(x, t) \text { on } \Omega \times R, & \nu>0, \\ u(x, t+\omega)=u(x, t) \text { for }(x, t) \in \Omega \times R \text { and }\left.u\right|_{\theta \Omega}=0 & \text { for } t \in R,\end{cases}
$$

where $f(x, t)$ is an $\omega$-periodic function in $t$ and $g(u)$ is a nonlinear function satisfying

$$
g(0)=0 \quad \text { and } \quad 0 \leqslant g^{\prime}(u) \leqslant k_{0}\left(1+|u|^{\alpha}\right)
$$

for some $\alpha \geqslant 0$. We assume $\nu=1$ without loss of generality.

Concerning the existence of a 'weak' solution of the problem (1.1) it is well known that if $f \in L^{2}\left(\omega ; L^{2}(\Omega)\right)$ and $0 \leqslant \alpha \leqslant 2 /(N-2)(0 \leqslant \alpha<\infty$ if $N=1,2)$, then the problem admits a solution $u$ in the class

$$
C\left(\omega ; \stackrel{\circ}{H}_{1}(\Omega)\right) \cap C^{1}\left(\omega ; L^{2}(\Omega)\right)
$$

(see Clements [1], Nakao [4], et cetera), where we denote by $C(\omega ; X), X$ : Banach space, the space of $\omega$-periodic continuous $X$-valued functions on $R=(-\infty,+\infty)$. Similar notations will be used freely.

Received 6 September 1991

Copyright Clearance Centre, Inc. Serial-fee code: 0004-9729/92 \$A2.00+0.00. 
Concerning the uniqueness and regularity, however, there remain some important open problems. First of all the uniqueness of weak solutions in the class (1.3) is not known. Moreover, it is not known whether the solution is classical or not for the case $\alpha=2 /(N-2)(N \geqslant 3)$, which is different from the case of initial-boundary value problems (see Sather [7], Wahl [9], et cetera). Of course, if $0<\alpha<2 /(N-2)$ the regularity problem becomes easier and has been solved (see Kato and Nakao [2]).

Our purpose in this paper is to discuss the uniqueness of the weak solution for the case $0 \leqslant \alpha \leqslant 2 /(N-2)$ as well as the regularity of it for the 'critical' case $\alpha=$ $2 /(N-2)$ in some restricted situations.

The problem we consider is in fact:

$$
\left\{\begin{array}{l}
\varepsilon u_{t t}+A u+u_{t}+g(u)=f(x, t) \quad \text { on } \Omega \times R, \quad \varepsilon>0, \\
u(x, t+\omega)=u(x, t) \quad \text { and }\left.\quad D^{\mu} u\right|_{\theta \Omega}=0, \quad 0 \leqslant|\mu| \leqslant m-1,
\end{array}\right.
$$

where $A$ is a symmetric uniformly elliptic operator of order $2 m$ :

$$
A u=\sum_{\substack{0 \leqslant|\alpha| \leqslant m \\ 0 \leqslant|\beta| \leqslant m}}(-1)^{|\alpha|} D^{\alpha}\left(a_{\alpha \beta}(x) D^{\beta} u\right)
$$

with smooth coefficients $a_{\alpha \beta}(x)\left(a_{\alpha \beta} \in C^{3 m}\right.$ is sufficient), and hence

$$
C\|u\|_{\stackrel{\circ}{H}_{m}}^{2} \leqslant(A u, u) \leqslant C^{\prime}\|u\|_{\stackrel{\circ}{H}_{m}}^{2} \quad \text { for } u \in \stackrel{\circ}{H}_{m} \cap H_{2 m}
$$

with some positive constants $C$ and $C^{\prime}$. Observe that if $\varepsilon=0$, the problem (1.4) is of parabolic type and for this equation the uniqueness is trivial and the regularity is easier. Motivated by these observations we shall investigate the uniqueness and the regularity problems for (1.4) under a smallness assumption on $\varepsilon>0$. Moreover we shall derive relations between the solutions $u_{\varepsilon}$ of (1.4) and the solution $u_{0}$ of the reduced parabolic problem:

$$
u_{t}+A u+g(u)=f(x, t) \quad \text { on } \Omega \times R
$$

with the periodicity and the boundary conditions.

When we treat the problem (1.4) with small parameter $\varepsilon$ the equation is sometimes called the 'hyperbolic parabolic' type. Such an equation has been considered by several authors; in particular our problem is closely related to that in Section 3, Chapter 4, in the book by Vejvoda [8]. In [8], however, the nonlinear term $g(u)$ of the equation is replaced by $\mu g(u)$ with a small parameter $\mu$ and essentially only small amplitude solutions are considered. There a Fourier expansion method is employed, while here we use an energy method. 
Recently, Milani [3] treated a similar problem for the quasilinear wave equation. But, in [3], it is assumed that the forcing term $f$ is small and the solutions treated are 'small', while we would emphasise again that we make no smallness assumption on $f$ and hence our solution may be 'large'. For other related works see Rabinowitz [6], Wahl $[10]$ and the references cited in the book [8].

\section{Statements of the Results}

The function spaces we use are all familiar and the definition of them will be omitted. But, we note that $\|\cdot\|$ denotes $L^{2}$-norm on $\Omega$. We also assume $0<\varepsilon \leqslant 1$ without loss of generality. Our first result reads as follows.

Theorem 1. Suppose that $f \in L^{2}\left(\omega ; L^{2}(\Omega)\right)$ and set

$$
d_{0} \equiv\left(\int_{0}^{\omega}\|f(t)\|^{2} d t\right)^{1 / 2}
$$

Concerning the nonlinear term we assume that $g$ belongs to $C^{1}(R)$ and satisfies

$$
g(0)=0 \quad \text { and } \quad 0 \leqslant g^{\prime}(u) \leqslant k_{0}\left(1+|u|^{\alpha}\right)
$$

for some $0 \leqslant \alpha \leqslant 2 m /(N-2 m)(0 \leqslant \alpha<\infty$ if $1 \leqslant N \leqslant 2 m)$.

Then, there exists a constant $C_{0}$ independent of $f$ and $g$ such that if

$$
\lambda\left(\varepsilon, d_{0}\right) \equiv C_{0} k_{0}^{2}\left(1+d_{0}^{2 \alpha}\right) \varepsilon<1,
$$

the problem (1.4) has a unique solution $u$ in the class

$$
C\left(\omega ; \stackrel{\circ}{H}_{m}(\Omega)\right) \cap C^{1}\left(\omega ; L^{2}(\Omega)\right)
$$

As a special case of Theorem 1 we have:

CoROLlary 1. If $g(u)$ is at most of linear growth, that is, (2.2) is satisfied with $\alpha=0$, there exists a constant $C_{1}$ independent of $g$ and $f$ such that if

$$
0<\varepsilon \leqslant C_{1} / k_{0}^{2}
$$

the solution of (1.4) is unique in the class (2.4).

The solution $u \equiv u_{\varepsilon}$ of the problem (1.4) converges to the solution $u_{0}$ of the parabolic problem (1.5) as $\varepsilon \rightarrow 0$ in the following sense: 
THEOREM 2. Under the assumption (2.3) we let $u_{\varepsilon}$ and $u_{0}$ be the solutions of (1.4) and (1.5), respectively, and set $w_{\epsilon}(t) \equiv u_{\epsilon}(t)-u_{0}(t)$. Then, there exists a constant $C_{2} \equiv C_{2}\left(d_{0}\right)$ such that

$$
\int_{0}^{\omega}\left\|w_{\varepsilon}(t)\right\|_{\dot{H}_{m}}^{2} d t \leqslant C_{2} \varepsilon
$$

If we assume, in addition, $f \in W^{1,2}\left(\omega ; L^{2}(\Omega)\right)$ and set

$$
d_{1} \equiv\left(\int_{0}^{\omega}\left\|f_{t}(t)\right\|^{2} d t\right)^{1 / 2}
$$

the solution $u_{e}(t)$ belongs to

$$
W^{2,2}\left(\omega ; L^{2}(\Omega)\right) \cap W^{1,2}\left(\omega ; \stackrel{\circ}{H}_{m}\right) \cap L^{2}\left(\omega ; H_{2 m}\right)
$$

and the estimate:

$$
\int_{0}^{\omega}\left\{\left\|\frac{\partial}{\partial t} w_{\varepsilon}(t)\right\|^{2}+\left\|w_{\varepsilon}(t)\right\|_{\dot{H}_{m}}^{2}+\left\|w_{\varepsilon}(t)\right\|_{H_{2 m}}^{2}\right\} d t \leqslant C_{3} \varepsilon^{2}
$$

holds, where $C_{3}$ is a constant depending on $d_{0}$ and $d_{1}$.

Next, we shall state our result concerning the regularity of the solution of (1.4).

ThEOREM 3. Let $2 m<N<4 m$. Suppose that $f$ belongs to

$$
W^{3,2}\left(\omega ; L^{2}(\Omega)\right) \cap C^{1}\left(\omega ; H_{m}\right) \cap C\left(\omega ; H_{2 m}\right)
$$

and set

$$
d_{i} \equiv\left(\int_{0}^{\omega}\left\|\left(\frac{\partial}{\partial t}\right)^{i} f(t)\right\|^{2} d t\right)^{1 / 2} \quad(i=0,1,2,3)
$$

$$
M_{1} \equiv \sup _{t}\left\|D_{t} f(t)\right\| \quad \text { and } \quad M_{2} \equiv \sup _{t}\left\{\|f(t)\|_{H_{2 m}}+\left\|f_{t}(t)\right\|_{H_{m}}\right\} .
$$

Concerning $g(u)$, suppose that $g$ belongs to $C^{2 m+1}(R)$ and satisfies, in addition to $(1.2)$,

$$
\left|g^{(i)}(u)\right| \leqslant k_{0}\left(1+|u|^{\alpha+1-i}\right), \quad i=1,2,
$$

with $\alpha=2 m /(N-2 m)$.

Then, under the assumption (2.3), the solution $u$ of Theorem 1 belongs in fact to

$$
C^{4}\left(\omega ; L^{2}(\Omega)\right) \bigcap_{j=0}^{s} C^{j}\left(\omega ; \stackrel{\circ}{H}_{m} \cap H_{4 m-j m}\right)
$$


and the following estimate holds:

$$
\begin{aligned}
& \int_{0}^{\omega}\left\|\left(\frac{\partial}{\partial t}\right)^{4} u(t)\right\|^{2} \\
& +\sup _{t}\left\{\varepsilon\left\|\left(\frac{\partial}{\partial t}\right)^{4} u(t)\right\|^{2}+\sum_{j=0}^{3}\left\|\left(\frac{\partial}{\partial t}\right)^{j} u(t)\right\|_{H_{4 m-j m}}^{2}\right\} \leqslant C_{4}<\infty
\end{aligned}
$$

where $C_{4}$ is a constant depending on $d_{i}(0 \leqslant i \leqslant 3)$ and $M_{i}(i=1,2)$, but independent of $\varepsilon$.

REMARK 1. By the Sobolev's imbedding theorem: $H_{2 m} \subset C(\Omega)$, we see $u \in$ $C\left(\omega ; C^{2 m}(\bar{\Omega})\right) \cap C^{1}\left(\omega ; C^{m}(\bar{\Omega})\right) \cap C^{2}(\omega ; C(\bar{\Omega}))$, that is, our solution is classical.

REMARK 2. The assumption $N<4 m$ is made for simplicity. We could treat the case $N \geqslant 4 m$ by carrying out a more careful analysis.

Corollary 2. Under the assumptions on $f$ and $g$ in Theorem 3 the parabolic problem (1.5) has a unique solution $u_{0}$ in the class

$$
W^{4,2}\left(\omega ; L^{2}(\Omega)\right) \bigcap_{j=0}^{3} C^{j}\left(\omega ; \stackrel{\circ}{H}_{m} H_{4 m-j m}\right)
$$

and the estimate

$$
\int_{0}^{\omega}\left\|\left(\frac{\partial}{\partial t}\right)^{4} u_{0}(t)\right\|^{2} d t+\sum_{j=0}^{3}\left\|\left(\frac{\partial}{\partial t}\right)^{j} u_{0}(t)\right\|_{H_{4 m-j m}}^{2} \leqslant C_{4}<\infty
$$

holds.

TheOREM 4. Let $u_{\varepsilon}(t)$ and $u_{0}(t)$ be the solutions of the problem (1.4) and (1.5), respectively, and set $w=u_{e}-u_{0}$. Then, under the assumptions of Theorem 3 the following estimates hold:

$$
\begin{gathered}
\sup _{t}\left\{\varepsilon\left\|w_{t}(t)\right\|^{2}+\|w(t)\|_{\dot{H}_{m}}^{2}\right\} \leqslant C\left(d_{0}, d_{1}\right) \varepsilon^{2} \\
\int_{0}^{\omega}\left\{\left\|w_{t t}(t)\right\|^{2}+\|w(t)\|_{H_{2 m}}^{2}\right\} d t+\sup _{t}\left\{\varepsilon\left\|w_{t t}(t)\right\|^{2}+\left\|w_{t}(t)\right\|_{H_{m}}^{2}\right\} \\
\leqslant C\left(d_{0}, d_{1}, d_{2}\right) \varepsilon^{2} \\
\int_{0}^{\omega}\left\{\left\|\left(\frac{\partial}{\partial t}\right)^{3} w(t)\right\|^{2}+\left\|w_{t}(t)\right\|_{H_{2 m}}^{2}\right\} d t
\end{gathered}
$$




$$
\begin{gathered}
+\sup _{t}\left\{\varepsilon\left\|\left(\frac{\partial}{\partial t}\right)^{3} w(t)\right\|^{2}+\left\|w_{t t}(t)\right\|_{H_{m}}^{2}\right\} \leqslant C\left(d_{0}, d_{1}, d_{2}, d_{3}\right) \varepsilon^{2}, \\
\int_{0}^{\omega}\left\|w_{t t}(t)\right\|_{H_{2 m}}^{2} d t \leqslant C\left(d_{0}, d_{1}, d_{2}, d_{3}\right) \varepsilon^{2} \\
\sup _{t}\left\{\|w(t)\|_{H_{4 m}}^{2}+\left\|w_{t}(t)\right\|_{H_{3 m}}^{2}\right\} \leqslant C\left(d_{0}, d_{1}, d_{2}, d_{3}, M_{1}, M_{2}\right) \varepsilon^{2}
\end{gathered}
$$

and

$$
\sup _{t}\left\|w_{t t}\right\|_{H_{2 m}} \leqslant C\left(d_{0}, d_{1}, d_{2}, d_{3}\right) \sqrt{\varepsilon}
$$

REMARK 3. When $1 \leqslant N \leqslant 2 m$, the assertions of Theorem 3 and Theorem 4 are valid without the smallness condition (2.3) on $\varepsilon$.

\section{Proof of Theorem 1}

First we shall prepare the following a priori estimates, which are in fact sufficient for the proof of the existence of a weak solution. In what follows we denote by $C$ general constants independent of $\varepsilon$ which may be different from line to line. To clarify the dependence on some quantity $q$ we use the notation $C(q)$ et cetera.

Proposition 3.1. Let $u(t)$ be a solution of (1.4) in the class (2.4). Then we have

$$
\int_{0}^{\omega}\left\|u_{t}(t)\right\|^{2} d t \leqslant d_{0}^{2}
$$

and

$$
\sup _{t}\left\{\varepsilon\left\|u_{t}(t)\right\|^{2}+\left\|A^{1 / 2} u(t)\right\|^{2}\right\} \leqslant C d_{0}^{2}
$$

where $\left\|A^{1 / 2} u\right\|$ is defined by

$$
\left\|A^{1 / 2} u\right\|=\left(\int_{\substack{\Omega_{0} \leqslant|\alpha| \leqslant m \\ 0 \leqslant|\beta| \leqslant m}} a_{\alpha \beta} D^{\alpha} u D^{\beta} u d x\right)^{1 / 2}
$$

which is equivalent to $\|u\|_{\dot{H}_{m}}$.

Proor: The proof is given by a standard energy method and we sketch it briefly. Multiplying the equation (1.4) by $u_{t}$ by $u_{t}$ and integrating over $[0, \omega] \times \Omega$ we have

$$
\int_{0}^{\omega}\left\|u_{t}(t)\right\|^{2} d t=\int_{0}^{\omega} \int_{\Omega} u_{t} f d x d t \leqslant\left(\int_{0}^{\omega}\left\|u_{t}\right\|^{2} d t\right)^{1 / 2}\left(\int_{0}^{\omega}\|f(t)\|^{2} d t\right)^{1 / 2}
$$


which implies (3.1) immediately. Next, multiplying the equation (1.4) by $u$ and integrating we get

$$
\int_{0}^{\omega}\left\{\left\|A^{i / 2} u(t)\right\|^{2}+\int_{\Omega} g(u) u d x\right\} d t=\varepsilon \int_{0}^{\omega}\left\|u_{t}(t)\right\|^{2} d t+\int_{0}^{\omega} \int_{\Omega} f u d x d t
$$

and hence

$$
\int_{0}^{\omega}\left\{\left\|A^{1 / 2} u(t)\right\|^{2}+\int_{\Omega} g(u) u d x\right\} d t \leqslant C d_{0}^{2} .
$$

From (3.1) and (3.5) we see

$$
\int_{0}^{\omega}\left\{\varepsilon\left\|u_{t}(t)\right\|^{2}+\left\|A^{1 / 2} u(t)\right\|^{2}+\int_{\Omega} g(u) u d x\right\} d t \leqslant C d_{0}^{2}
$$

and hence, there exists $t^{*} \in(0, \omega)$ such that

$$
\varepsilon\left\|u_{t}\left(t^{*}\right)\right\|^{2}+\left\|A^{1 / 2} u\left(t^{*}\right)\right\|^{2}+\int_{\Omega} g\left(u\left(t^{*}\right)\right) u\left(t^{*}\right) d x \leqslant C d_{0}^{2} .
$$

Thus, using the equation (1.4), we see

$$
\begin{aligned}
\sup _{t}\left\{\frac{1}{2}\left(\varepsilon\left\|u_{t}(t)\right\|^{2}+\left\|A^{1 / 2} u(t)\right\|^{2}\right)\right. & \left.+\int_{\Omega} \int_{0}^{u(t)} g(\eta) d \eta d x\right\} \\
\leqslant \frac{1}{2}\left(\varepsilon\left\|u_{t}\left(t^{*}\right)\right\|^{2}+\left\|A^{1 / 2} u\left(t^{*}\right)\right\|^{2}\right) & +\int_{\Omega} \int_{0}^{u\left(t^{*}\right)} g(\eta) d \eta d x \\
& +\int_{0}^{\omega} \int_{\Omega}\left|f u_{t}\right| d x d t \leqslant C d_{0}^{2}
\end{aligned}
$$

which completes the proof of (3.2)

Theorem 1 is an immediate consequence of the following proposition.

Proposition 3.2. Letting $u$ and $v$ be two solutions of the problem (1.4) in the class (2.4), we have the estimate for $w=u-v$ :

$$
\int_{0}^{\omega}\left\|A^{1 / 2} w(t)\right\|^{2} d t \leqslant \lambda\left(\varepsilon, d_{0}\right) \int_{0}^{\omega}\left\|A^{1 / 2} w(t)\right\|^{2} d t
$$

where $\lambda\left(\varepsilon, d_{0}\right)$ is the constant given by (2.3).

Proof: $w=u-v$ satisfies the equation

$$
\varepsilon w_{t t}+A w+w_{t}+g(u)+g(v)=0
$$


together with the periodicity and the boundary conditions. Multiplying the equation (3.9) by $w$ and integrating we have

$$
\int_{0}^{\omega}\left\|A^{1 / 2} w(t)\right\|^{2} d t \leqslant \varepsilon \int_{0}^{\omega}\left\|w_{t}(t)\right\|^{2} d t
$$

where the monotonicity of $g(u)$ is used essentially. Next, multiplying the equation by $w_{t}$ and integrating we have

$$
\begin{aligned}
& \int_{0}^{\omega}\left\|w_{t}(t)\right\|^{2} d t=-\int_{0}^{\omega} \int_{n}(g(u)-g(v)) w_{t} d x d t \\
& \leqslant k_{0}\left\{\int_{0}^{\omega} \int_{\Omega}\left\{1+(|u|+|v|)^{\alpha}\right\}^{2}|w|^{2} d x d t\right\}^{1 / 2}\left(\int_{0}^{\omega}\left\|w_{t}\right\|^{2} d t\right)^{1 / 2} \\
& \quad \leqslant C k_{0}\left\{\int_{0}^{\omega}\left(1+\left\|A^{1 / 2} u\right\|+\left\|A^{1 / 2} v\right\|\right)^{2 \alpha}\left\|A^{1 / 2} w\right\|^{2} d t\right\}^{1 / 2}\left(\int_{0}^{\omega}\left\|w_{t}\right\|^{2} d t\right)^{1 / 2},
\end{aligned}
$$

where we have used the Sobolev inequality:

$$
\begin{gathered}
\|u\|_{2(\alpha+1)} \leqslant C\left\|A^{1 / 2} u\right\| \text { for } u \in \stackrel{\circ}{H}_{m} \text { if } 0 \leqslant \alpha \leqslant 2 m /(N-2 m), \\
(0 \leqslant \alpha<\infty \text { if } 1 \leqslant N \leqslant 2 m) .
\end{gathered}
$$

It follows from (3.10), (3.11) and (3.7) that

$$
\int_{0}^{\omega}\left\|A^{1 / 2} w(t)\right\|^{2} d t \leqslant C \epsilon k_{0}^{2}\left(1+d_{0}^{2 \alpha}\right) \int_{0}^{\omega}\left\|A^{1 / 2} w\right\|^{2} d t .
$$

\section{Proof of Theorem 2}

For $w_{\varepsilon}=u_{\varepsilon}-u_{0}$ we have the equation:

$$
\varepsilon u_{t t}+A w+w_{t}+g(u)-g\left(u_{0}\right)=0 \quad\left(u \equiv u_{\varepsilon}, w \equiv w_{\epsilon}\right)
$$

Multiplying the equation (4.1) by $w$ and using the monotonicity of $g(u)$ and the periodicity of $u$ and $w$ we see

$$
\begin{aligned}
\int_{0}^{\omega}\left\|A^{1 / 2} w(t)\right\| d t & \leqslant-\varepsilon \int_{0}^{\omega} \int_{\Omega} u_{t t} w d x d t \\
& =\varepsilon \int_{0}^{\omega} \int_{\Omega} u_{t} w_{t} d x d t \\
& \leqslant \varepsilon\left(\int_{0}^{\omega}\left\|u_{t}(t)\right\|^{2} d t\right)^{1 / 2}\left(\int_{0}^{\omega}\left\|w_{t}(t)\right\|^{2} d t\right)^{1 / 2} \\
& \leqslant \varepsilon d_{0}\left(\int_{0}^{\omega}\left\|w_{t}(t)\right\|^{2} d t\right)^{1 / 2} \text { (by (3.1)). }
\end{aligned}
$$


Moreover, we see easily

$$
\begin{aligned}
\left(\int_{0}^{\omega}\left\|w_{t}(t)\right\|^{2} d t\right)^{1 / 2} & \leqslant\left(\int_{0}^{\omega}\left\|u_{t}(t)\right\|^{2} d t\right)^{1 / 2}+\left(\int_{0}^{\omega}\left\|u_{0 t}(t)\right\|^{2} d t\right)^{1 / 2} \\
& \leqslant C d_{0}
\end{aligned}
$$

and hence, by (4.2)

$$
\int_{0}^{\omega}\left\|A^{1 / 2} w(t)\right\|^{2} d t \leqslant C d_{0}^{2} \varepsilon
$$

which is the first assertion of Theorem 2 .

Next, for the proof of second part, we assume $f \in W^{1 / 2}\left(\omega ; L^{2}(\Omega)\right)$.

Then, we shall use the equation:

$$
\varepsilon u_{t t t}+A u_{t}+u_{t t}+g^{\prime}(u) u_{t}=f_{t}
$$

to derive further a priori estimates. In fact, the following proposition assures us that the solution $u \equiv u_{\varepsilon}$ belongs to

$$
C^{2}\left(\omega ; L^{2}(\Omega)\right) \cap C^{1}\left(\omega ; \stackrel{\circ}{H}_{m}\right) \cap C\left(\omega ; H_{2 m} \cap \stackrel{\circ}{H}_{m}\right)
$$

PROPOSITION 4.1. Under the assumption (2.3), we have the estimates

$$
\int_{0}^{\omega}\left\|u_{t t}(t)\right\|^{2} d t \leqslant C\left\{d_{1}^{2}+k_{0}^{2}\left(1+d_{0}^{2 \alpha}\right) d_{0} d_{1}\right\}
$$

and

$$
\sup _{t}\left\{\varepsilon\left\|u_{t t}(t)\right\|^{2}+\left\|A^{1 / 2} u_{t}(t)\right\|^{2}\right\} \leqslant C d_{1}^{2}
$$

Proof: Multiplying the equation (4.3) by $u_{t t}$ and integrating we see easily

$$
\begin{aligned}
\int_{0}^{\omega}\left\|u_{t t}(t)\right\|^{2} d t & \leqslant d_{1}^{2}+2 \int_{0}^{\omega} \int_{\Omega}\left|g^{\prime}(u)\left\|u_{t}\right\| U_{t t}\right| d x d t \\
& \leqslant d_{1}^{2}+2 k_{0} \int_{0}^{\omega} \int_{\Omega}\left(1+|u|^{\alpha}\right)\left|u_{t}\right|\left|u_{t t}\right| d x d t \\
& \leqslant d_{1}^{2}+C k_{0}^{2} \int_{0}^{\omega}\left(1+\left\|A^{1 / 2} u(t)\right\|^{2 \alpha}\right)\left\|A^{1 / 2} u_{t}(t)\right\|^{2} d t \\
& +\frac{1}{2} \int_{0}^{\omega}\left\|u_{t t}(t)\right\|^{2} d t
\end{aligned}
$$


and hence, with the aid of (3.2),

$$
\int_{0}^{\omega}\left\|u_{t t}(t)\right\|^{2} d t \leqslant 2 d_{1}^{2}+C k_{0}^{2}\left(1+d_{0}^{2 \alpha}\right) \int_{0}^{\omega}\left\|A^{1 / 2} u_{t}(t)\right\|^{2} d t
$$

On the other hand, multiplying the equation (4.3) by $u_{t}$ and integrating we see

$$
\begin{gathered}
\int_{0}^{\omega}\left\|A^{1 / 2} u_{t}(t)\right\|^{2} d t \leqslant \varepsilon \int_{0}^{\omega}\left\|u_{t t}(t)\right\|^{2} d t+\int_{0}^{\omega} \int_{\Omega} f_{t} u_{t} d x d t \\
\leqslant \varepsilon \int_{0}^{\omega}\left\|u_{t t}(t)\right\|^{2} d t+\frac{1}{2} \int_{0}^{\omega}\left\|A^{1 / 2} u_{t}(t)\right\|^{2} d t+C d_{1}^{2} .
\end{gathered}
$$

It follows from (4.6) and (4.7) that

$$
\begin{aligned}
& \int_{0}^{\omega}\left\|u_{t t}(t)\right\|^{2} d t \\
& \quad \leqslant C k_{0}^{2}\left(1+d_{0}^{2 \alpha}\right) \varepsilon \int_{0}^{\omega}\left\|u_{t t}(t)\right\|^{2} d t+2 d_{1}^{2}+C k_{0}^{2}\left(1+d_{0}^{2 \alpha}\right) d_{0} d_{1} .
\end{aligned}
$$

Thus, under the assumption $C k_{0}^{2}\left(1+d_{0}^{2 \alpha}\right) \varepsilon<1 / 2$, which is equivalent to (2.3) by changing $C_{0}$ if necessary, we have the estimate (4.4).

Moreover, (4.5) follows immediately from (4.4) and (4.7). Finally we note that these estimates give

$$
\int_{0}^{\omega}\left\{\varepsilon\left\|u_{t t}(t)\right\|^{2}+\left\|A^{1 / 2} u_{t}(t)\right\|^{2}\right\} d t \leqslant C d_{1}^{2} \quad(0<\varepsilon \leqslant 1)
$$

which implies, as in the proof of (3.2), the estimate (4.5).

Now, we shall prove the second part of Theorem 2.

Multiplying the equation (4.1) by $w(t) \equiv u_{\varepsilon}-u_{0}$ and integrating over $\Omega \times[0, \omega]$ as is usual, we have

$$
\begin{aligned}
\int_{0}^{\omega}\left\|A^{1 / 2} w(t)\right\|^{2} d t & \leqslant-\varepsilon \int_{0}^{\omega} \int_{\Omega} u_{t t} w d x d t \\
& \leqslant C \varepsilon\left(\int_{0}^{\omega}\left\|u_{t t}(t)\right\|^{2} d t\right)^{1 / 2}\left(\int_{0}^{\omega}\left\|A^{1 / 2} w(t)\right\|^{2} d t\right)^{1 / 2}
\end{aligned}
$$

and hence, by (4.4)

$$
\int_{0}^{\omega}\left\|A^{1 / 2} w(t)\right\|^{2} d t \leqslant C\left(d_{0}, d_{1}\right) \varepsilon^{2}
$$


Further, multiplying the equation (4.1) by $w_{t}$ we see

$$
\begin{aligned}
\int_{0}^{\omega}\left\|w_{t}(t)\right\|^{2} d t \leqslant & -\varepsilon \int_{0}^{\omega} \int_{\Omega} u_{t t} w_{t} d x d t \\
& +C k_{0} \int_{0}^{\omega} \int_{\Omega}\left(1+|u|^{\alpha}+\left|u_{0}\right|^{\alpha}\right)|w|\left|w_{t}\right| d x d t \\
\leqslant & C\left(d_{0}, d_{1}\right)\left\{\varepsilon\left(\int_{0}^{\omega}\left\|u_{t t}(t)\right\|^{2} d t\right)^{1 / 2}+\left(\int_{0}^{\omega}\left\|A^{1 / 2} w(t)\right\|^{2} d t\right)^{1 / 2}\right\} \\
& \times\left(\int_{0}^{\omega}\left\|w_{t}(t)\right\|^{2} d t\right)^{1 / 2}
\end{aligned}
$$

and we conclude from (2.6) and (4.4) that

$$
\int_{0}^{\omega}\left\|w_{t}(t)\right\|^{2} d t \leqslant C\left(d_{0}, d_{1}\right) \varepsilon^{2}
$$

Similarly, multiplying the equation (4.1) by $A w$ and integrating, we can prove

$$
\int_{0}^{\omega}\|A w(t)\|^{2} d t \leqslant C\left(d_{0}, d_{1}\right) \varepsilon^{2}
$$

The proof of Theorem 2 is now complete.

\section{Proofs of Theorem 3 and Theorem 4}

By standard arguments it suffices for the proof of Theorem 3 to derive the a priori estimate (2.13) for an assumed solution $u$ in the class (2.12). For this we observe:

PROPOSITION 5.1. Let $u(t)$ be the solution of (1.4) in the sense of Theorem 1 and let $U(t)$ be a solution in the class $C^{1}\left(\omega ; L^{2}(\Omega)\right) \cap C\left(\omega ; \stackrel{\circ}{H}_{m}\right)$ of the problem

$$
\left\{\begin{array}{l}
\varepsilon U_{t t}+A U+U_{t}+g^{\prime}(u) U=F(x, t) \quad \text { on } \Omega \times R \\
U(x, t+\omega)=U(x, t) \quad \text { and }\left.\quad U\right|_{\theta n}=0
\end{array}\right.
$$

with $F \in L^{2}\left(\omega ; L^{2}(\Omega)\right)$.

Then, under the assumption (2.3), the estimates

$$
\begin{aligned}
& \int_{0}^{\omega}\left\|U_{t}(t)\right\|^{2} d t \leqslant C\left\{1+k_{0}^{2}\left(1+d_{0}^{2 \alpha}\right)\right\} \int_{0}^{\omega}\|F(t)\|^{2} d t \\
& \sup _{t}\left\{\varepsilon\left\|U_{t}(t)\right\|^{2}+\left\|A^{1 / 2} U(t)\right\|^{2}\right\} \leqslant C \int_{0}^{\omega}\|F(t)\|^{2} d t
\end{aligned}
$$

and

hold. 
Proof: The proof is essentially the same as that of (4.5) in Proposition 4.1 and is omitted.

Using Proposition 5.1 and Proposition 4.1 we shall show:

Proposition 5.2. Under the assumption (2.3) the solution $u(t)$ in the class (2.12) satisfies

$$
\begin{aligned}
& \int_{0}^{\omega}\left\|u_{t t t}(t)\right\|^{2} d t \leqslant C_{5}\left(d_{0}, d_{1}, d_{2}\right)<\infty \\
& \sup _{t}\left\{\varepsilon\left\|u_{t t t}(t)\right\|^{2}+\left\|A^{1 / 2} u_{t t}(t)\right\|^{2} \leqslant C_{6}\left(d_{0}, d_{1}, d_{2}\right)<\infty,\right.
\end{aligned}
$$

and

$$
\sup _{t}\left\|A u_{t}(t)\right\| \leqslant C_{7}\left(d_{0}, d_{1}, d_{2}\right)<\infty
$$

for some constants $C_{5}, C_{6}, C_{7}$ depending on the quantities indicated but independent of $\varepsilon$.

ProOF: Setting $U=u_{t t}, U$ satisfies the equation

$$
\varepsilon U_{t t}+A U+U_{t}+g^{\prime}(u) U=-g^{\prime \prime}(u)\left(u_{t}\right)^{2}+f_{t t}
$$

with the periodicity and the boundary conditions. Here,

$$
\begin{aligned}
\int_{\Omega}\left|g^{\prime \prime}(u)\right|^{2}\left|u_{t}\right|^{4} d x & \leqslant k_{0}^{2} \int_{\Omega}\left(1+|u|^{\alpha-1}\right)^{2}\left|u_{t}\right|^{4} d x \\
& \leqslant C k_{0}^{2}\left(1+\left\|A^{1 / 2} u\right\|^{2 \alpha-2}\right)\left\|A^{1 / 2} u_{t}\right\|^{4} \\
& \leqslant C k_{0}^{2}\left(1+d_{0}^{2 \alpha-2}\right) d_{1}^{4} \quad \text { (by (3.2) and (4.5)). }
\end{aligned}
$$

Thus, applying Proposition 5.1 we obtain the estimates (5.3) and (5.4). Moreover, returning to the equation (4.3) we see easily

$$
\begin{aligned}
\left\|A u_{t}(t)\right\| & \leqslant C\left\{\varepsilon\left\|u_{t t t}(t)\right\|+\left\|u_{t t}(t)\right\|+\left(\int_{\Omega}\left|g^{\prime}(u) u_{t}\right|^{2} d x\right)^{1 / 2}+\left\|f_{t}(t)\right\|\right\} \\
& \leqslant C_{7}\left(d_{0}, d_{1}, d_{2}, M_{1}\right)<\infty
\end{aligned}
$$

for some constant $C_{7}$.

PROPOSITION 5.3. Under the assumption (2.3), the solution $u(t)$ in the class 
(2.12) satisfies

$$
\begin{gathered}
\int_{0}^{\omega}\left\|\left(\frac{\partial}{\partial t}\right)^{4} u(t)\right\|^{2} d t \leqslant C_{8}\left(d_{0}, d_{1}, d_{2}, d_{3}\right)<\infty \\
\sup _{t}\left\{\varepsilon\left\|\left(\frac{\partial}{\partial t}\right)^{4} u(t)\right\|^{2}+\left\|A^{1 / 2} u_{t t t}(t)\right\|^{2}\right\} \leqslant C_{9}\left(d_{0}, d_{1}, d_{2}, d_{3}\right)<\infty
\end{gathered}
$$

and

$$
\sup _{t}\left\|A u_{t t}(t)\right\| \leqslant C_{10}\left(d_{0}, d_{1}, d_{2}, d_{3}\right)<\infty .
$$

Proof: Setting $U=u_{t t t}$, it satisfies the equation

$$
U_{t t}+A U+U_{t}+g^{\prime}(u) U=-3 g^{\prime \prime}(u) u_{t} u_{t t}-g^{\prime \prime \prime}(u)\left(u_{t}\right)^{3}+f_{t t t}
$$

with the periodicity and the boundary conditions. Here,

$$
\begin{aligned}
\int_{\Omega}\left|g^{\prime \prime}(u) u_{t} u_{t t}\right|^{2} d x & \leqslant k_{0}^{2} \int_{\Omega}\left(1+|u|^{\alpha-2}\right)^{2}\left|u_{t}\right|^{2}\left|u_{t t}\right|^{2} d x \\
& \leqslant C k_{0}^{2}\left(1+\left\|A^{1 / 2} u\right\|^{2 \alpha-4}\right)\left\|A^{1 / 2} u_{t}\right\|^{2}\left\|A^{1 / 2} u_{t t}\right\|^{2} \\
& \leqslant C\left(d_{0}, d_{1}, d_{2}\right)<\infty \quad \text { (by (3.2), (4.5) and (5.4)). }
\end{aligned}
$$

Thus, applying Proposition 5.1 to (5.10), we get (5.7) and (5.8). Furthermore, returning to the equation (5.6) and using the estimates in hand we see easily

$$
\begin{aligned}
\left\|A u_{t t}(t)\right\| & \leqslant\left\{\varepsilon\left\|\left(\frac{\partial}{\partial t}\right)^{4} u(t)\right\|+\left\|u_{t t t}(t)\right\|+\left\|g^{\prime}(u) u_{t t}\right\|+\left\|g^{\prime \prime}(u) u_{t}^{2}\right\|+\left\|f_{t t}\right\|\right\} \\
& \leqslant C\left(d_{0}, d_{1}, d_{2}, d_{3}\right)<\infty .
\end{aligned}
$$

(Note that $\sup _{t}\left\|f_{t t}(t)\right\| \leqslant C\left(d_{2}+d_{3}\right)$.)

It remains to derive estimates for $\|u(t)\|_{H_{4 m}}$ and $\left\|u_{t}(t)\right\|_{H_{3 m}}$.

Proposition 5.4. Under the assumption (2.3) the solution $u(t)$ in the class (2.12) satisfies further

$$
\|u(t)\|_{H_{4 m}} \leqslant C\left(d_{0}, d_{1}, d_{2}, d_{3}, M_{2}\right)<\infty
$$

and

$$
\left\|u_{t}(t)\right\|_{H_{3 m}} \leqslant C\left(d_{0}, d_{1}, d_{2}, d_{3}, M_{2}\right)<\infty
$$


Proof: To prove (5.11) we use the equation (1.4):

$$
A u=-\varepsilon u_{t t}-u_{t}-g(u)+f(t) .
$$

Here, we see by (5.5) and (5.9)

$$
\left\|-\varepsilon u_{t t}-u_{t}+f\right\|_{H_{2 m}} \leqslant C\left(d_{0}, d_{1}, d_{2}\right)+M_{2} .
$$

To estimate $\|g(u)\|_{H_{2 m}}$, we denote by $D^{k}$ any partial differentiations in $x=\left(x_{1}, x_{2}, \ldots x_{N}\right)$ of order $k$. The estimation is standard and we sketch it briefly. First, notice that

$$
D^{2 m} g(u(t))=\sum_{k=1}^{2 m} g^{(k)}(u) \sum_{\sigma \in S_{k}}(D u)^{\sigma_{1}}\left(D^{2} u\right)^{\sigma_{2}} \ldots\left(D^{2 m} u\right)^{\sigma_{2 m}}
$$

where we set

$$
S_{k} \equiv\left\{\begin{array}{l|l}
\sigma \equiv\left(\sigma_{1}, \sigma_{2}, \ldots, \sigma_{2 m}\right) \in N^{2 m} & \begin{array}{l}
\sigma_{1}+\sigma_{2}+\ldots+\sigma_{2 m}=k, \\
\sigma_{1}+2 \sigma_{2}+\ldots+2 m \sigma_{2 m}=2 m
\end{array}
\end{array}\right\} .
$$

We know that by the estimate (4.5) and (3.2)

$$
\|A u(t)\| \leqslant \varepsilon\left\|u_{t t}(t)\right\|+\left\|u_{t}(t)\right\|+\|g(u)\|+\|f(t)\| \leqslant C\left(d_{0}, d_{1}\right)<\infty
$$

and hence, by the assumption $N<4 m$,

$$
\|u(t)\|_{\infty} \leqslant C\|A u(t)\| \leqslant C\left(d_{0}, d_{1}\right)<\infty .
$$

Thus,

$$
\begin{aligned}
\left\|D^{2 m} g(u)\right\| & \leqslant C\left(d_{0}, d_{1}\right) \sum_{k=1}^{2 m} \sum_{S_{k}}\left\|(D u)^{\sigma_{1}}\left(D^{2} u\right)^{\sigma_{2}} \ldots\left(D^{2 m} u\right)^{\sigma_{2 m}}\right\| \\
& \leqslant C\left(d_{0}, d_{1}\right) \sum_{k=1}^{2 m} \sum_{S_{k}}\|D u\|_{p_{1} \sigma_{1}}^{\sigma_{1}}\left\|D^{2} u\right\|_{p_{2} \sigma_{2}}^{\sigma_{2}} \ldots\left\|D^{2 m} u\right\|_{p_{m} \sigma_{2 m}}^{\sigma_{2 m}} \\
& \leqslant C\left(d_{0}, d_{1}\right) \sum_{k=1}^{2 m}\|u\|_{H_{2 m}}^{k} \leqslant C\left(d_{0}, d_{1}\right)<\infty
\end{aligned}
$$

where we should choose $p_{j}(j=1,2, \ldots, 2 m)$ in such a way that

and

$$
\begin{gathered}
2 \leqslant p_{j} \leqslant \infty, \quad \sum_{j=1}^{2 m} \frac{1}{p_{j}}=\frac{1}{2} \\
p_{j} \begin{cases}\leqslant 2 N / \sigma_{j}(N-4 m+2 j) & \text { if } N>4 m-2 j, \\
<\infty & \text { if } N=4 m-2 j, \\
=\infty & \text { if } N<4 m-2 j .\end{cases}
\end{gathered}
$$


Such a choice of $\left\{p_{j}\right\}$ is possible, since

$$
\sum_{j=1}^{2 m} \frac{\sigma_{j}(N-4 m+2 j)}{2 N}<\frac{1}{2} \quad \text { if } \quad \sigma_{2 m}=0 .
$$

The estimate (5.11) follows from (5.13), (5.17) and the equation (1.4).

Next, using the equation (4.3):

$$
A u_{t}=-\varepsilon u_{t t t}-u_{t t}-g^{\prime}(u) u_{t}+f_{t}
$$

we can derive (5.12) by a similar argument, the details being omitted (see [5]).

Now, all the estimates required for the proof of Theorem 3 have been derived and the proof is complete.

\section{Proof of Theorem 4}

Setting $w=u_{\varepsilon}-u_{0}$, we have

$$
\varepsilon w_{t t}+A w+w_{t}+\int_{0}^{1} g^{\prime}\left(\theta u_{\varepsilon}+(1-\theta) u_{0}\right) d \theta w=-\varepsilon u_{0 t t} .
$$

Notice that the result of Proposition 5.1 is valid even if $g^{\prime}(u)$ in (5.1) is replaced by $\int_{0}^{1} g^{\prime}\left(\theta u_{\varepsilon}+(1-\theta) u_{0}\right) d \theta$. Thus, we obtain (2.15) immediately.

(Note that $\int_{0}^{\omega}\left\|u_{0 t t}\right\|^{2} d t \leqslant C\left(d_{1}\right)$.)

Differentiating the equation (5.18) with respect to $t$ we get

$$
\begin{aligned}
& \varepsilon w_{t t t}+A w_{t}+w_{t t}+\int_{0}^{1} g^{\prime}\left(\theta u_{e}+(1-\theta) u_{0}\right) d \theta w_{t} \\
& =-\int_{0}^{1} g^{\prime \prime}\left(\theta u_{\epsilon}+(1-\theta) u_{0}\right)\left(\theta u_{e t}+(1-\theta) u_{0 t}\right) d \theta w-\varepsilon u_{0 t t t} .
\end{aligned}
$$

Applying a variant of Proposition 5.1 to (5.19) we have (2.16). (See the proof of Proposition 5.2.) Moreover, differentiating the equation (5.19) once more we get

$$
\begin{aligned}
\varepsilon & \left(\frac{\partial}{\partial t}\right)^{4} w+A w_{t t}+w_{t t t}+\int_{0}^{1} g^{\prime}\left(\theta u_{e}+(1-\theta) u_{0}\right) d \theta w_{t t} \\
= & -\int_{0}^{1} g^{\prime \prime \prime}\left(\theta u_{\varepsilon}+(1-\theta) u_{0}\right)\left(\theta u_{\varepsilon t}+(1-\theta) u_{0 t}\right)^{2} d \theta w \\
& -\int_{0}^{1} g^{\prime \prime}\left(\theta u_{\varepsilon}+(1-\theta) u_{0}\right)\left(\theta u_{e t t}+(1-\theta) u_{0 t t}\right) d \theta w \\
& -2 \int_{0}^{1} g^{\prime \prime}\left(\theta u_{\varepsilon}+(1-\theta) u_{0}\right)\left(\theta u_{\varepsilon t}+(1-\theta) u_{0 t}\right) d \theta w_{t} \\
& -\varepsilon\left(\frac{\partial}{\partial t}\right)^{4} u_{0} .
\end{aligned}
$$


Applying a variant of Proposition 5.1 to (5.20) once more, and repeating similar estimations as in the proof of Proposition 5.3, we can prove (2.17) and (2.18). The estimate (2.19) follows from similar arguments as in the proof of Proposition 5.4, the details being omitted.

Finally, using the equation (5.20) we get

$$
\begin{aligned}
\sup _{t}\left\|A w_{t t}(t)\right\| \leqslant & \sup _{t}\left\{\left\|\varepsilon\left(\frac{\partial}{\partial t}\right)^{4} u(t)\right\|+\left\|w_{t t t}(t)\right\|\right\} \\
& +\sup _{t} \int_{0}^{1}\left|g^{\prime}\left(\theta u_{\epsilon}+(1-\theta) u_{0}\right)\right| d \theta\left\|w_{t t}(t)\right\| \\
& +\sup _{t} \int_{0}^{1}\left|g^{\prime \prime \prime}\left(\theta u_{\varepsilon}+(1-\theta) u_{0}\right)\left(\theta u_{e t}+(1-\theta) u_{0 t}\right)^{2}\right| d \theta\|w(t)\| \\
& +\sup _{0} \int_{0}^{1}\left|g^{\prime \prime}\left(\theta u_{\varepsilon}+(1-\theta) u_{0}\right)\left(\theta u_{e t t}+(1-\theta) u_{0 t t}\right)\right| d \theta\|w(t)\| \\
& +2 \sup _{t} \int_{0}^{1}\left|g^{\prime \prime}\left(\theta u_{\varepsilon}+(1-\theta) u_{0}\right)\left(\theta u_{e t}+(1-\theta) u_{0 t}\right)\right| d \theta\left\|w_{t}(t)\right\| \\
\leqslant & C\left(d_{0}, d_{1}, d_{2}, d_{3}\right) \sqrt{\varepsilon} \quad(\text { by }(5.8)),
\end{aligned}
$$

which proves (2.20). The proof is complete.

\section{REFERENCES}

[1] J.C. Clements, 'Existence theorem for some nonlinear equation of evolution', Canad. J. Math. 22 (1970), 726-745.

[2] H. Kato and M. Nakao, 'Existence of strong and smooth periodic solutions of some nonlinear evolution equations', Math. Rep. Kyushu Univ. 14 (1983), 57-88.

[3] A.J. Milani, 'Time periodic smooth solutions of hyperbolic quasi-linear equations with dissipation term and their approximation by parabolic equations', Ann. Mat. Pura Appl. 140 (1985), 331-344.

[4] M. Nakao, 'Bounded, periodic and almost periodic solutions of nonlinear hyperbolic partial differential equations', J. Differential Equations 23 (1977), 368-386.

[5] M. Nakao, 'Global existence of classical solutions to the initial-boundary value problem of the semilinear wave equations with a degenerate dissipative term', Nonlinear Anal. 14 (1990), 115-140.

[6] P.H. Rabinowitz, 'Periodic solutions of nonlinear hyperbolic partial differential equations', Comm. Pure. Appl. Math. 20 (1967), 145-205.

[7] J. Sather, 'The existence of a global classical solution of the initial-boundary value problem for $\square u+u^{3}=f '$, Arch. Rational Mech. Anal. 22 (1966), 292-307.

[8] O. Vejvoda, Partial differential equations: time-periodic solutions (Martinus Nijhoff Publishers, The Hague, Boston, London, 1982). 
[9] W. von Wahl, 'Klassische Lösungen nichtlinearer Wellengleichungen im Grossen', Math. Z. 112 (1969), 241-279.

[10] W. von Wahl, 'Periodic solutions of nonlinear wave equations with a dissipative term', Math. Ann. 190 (1971), 313-322.

Department of Mathematics

College of General Education

Kyushu University

Fukuoka 810

Japan 